\title{
Decay of metastable current states in one-dimensional resonant tunneling devices
}

\author{
O. A. Tretiakov, 1 , and K. A. Matveev ${ }^{2,1}$ \\ ${ }^{1}$ Department of Physics, Duke University, Durham, North Carolina 27708, USA \\ ${ }^{2}$ Materials Science Division, Argonne National Laboratory, Argonne, Illinois 60439, USA
}

(Dated: January 10, 2006)

\begin{abstract}
Current switching in a double-barrier resonant tunneling structure is studied in the regime where the current-voltage characteristic exhibits intrinsic bistability, so that in a certain range of bias two different steady states of current are possible. Near the upper boundary $V_{t h}$ of the bistable region the upper current state is metastable, and because of the shot noise it eventually decays to the stable lower current state. We find the time of this switching process in strip-shaped devices, with the width small compared to the length. As the bias $V$ is tuned away from the boundary value $V_{t h}$ of the bistable region, the mean switching time $\tau$ increases exponentially. We show that in long strips $\ln \tau \propto\left(V_{t h}-V\right)^{5 / 4}$, whereas in short strips $\ln \tau \propto\left(V_{t h}-V\right)^{3 / 2}$. The one-dimensional geometry of the problem enables us to obtain analytically exact expressions for both the exponential and the prefactor of $\tau$. Furthermore, we show that, depending on the parameters of the system, the switching can be initiated either inside the strip, or at its ends.
\end{abstract}

PACS numbers: 73.40.Gk, 73.21.Ac, 73.50.Td

\section{INTRODUCTION}

In the last two decades with advances of miniaturization techniques various resonant tunneling structures became the subject of intensive research. It was experimentally observed that the current-voltage characteristics of resonant tunneling devices, such as double-barrier resonant tunneling structures 1.2.3.4.5 (DBRTS) and superlattices,$\frac{6.7 .8 .9}{}$ exhibit intrinsic bistabilities. Namely, it was shown that for each value of bias in the bistable region of the $I-V$ curve the current can take two different values. A bistable $I-V$ curve of a double-barrier structure derived theoretically in Ref. 10 is depicted schematically in Fig. 11 It is theoretically established ${ }^{11.12}$ that near the boundary $V_{t h}$ of the bistable region (e.g., at point $\boldsymbol{A})$ the upper current state is metastable. In recent experiments 8.9 the switching from the metastable to stable current state was studied in superlattices. In particular, the mean switching time $\tau$ was measured.

The problems of decay of metastable states were studied theoretically in various fields, such as condensed matter physics, $13,14,15,16,17,18,19$ quantum field theory ${ }_{20.21 .22 .23 .24}^{20}$ and chemical kinetics ${ }^{25.26}$ In the context of resonant tunneling structures it was addressed in Refs. 11 12. A typical double-barrier resonant tunneling structure consists of three semiconducting layers of GaAs separated by two insulating layers of GaAlAs. In the narrow middle layer of GaAs the electron motion in the direction normal to the layers is quantized, so that a quantum well is formed. In the bistable region (Fig. 1) the two current states correspond to two different values of electron density $n$ in the well. The density $n$ exhibits shot noise fluctuations caused by random events of tunneling of electrons in and out of the well. Thus it becomes possible for the device to switch from one current state to the other. In Refs. 11 it was shown that there are two regimes of current switching. In the case of relatively small samples the electrons spread uniformly over the well due to diffusion. Then the switching occurs simultaneously in the entire area of the sample, and it was found that the switching time $\tau$ is exponentially large, with $\ln \tau \propto\left(V_{t h}-V\right)^{3 / 2}$. In larger samples the density in the well is not uniform, and the switching occurs through nucleation mechanism: it initiates in a small region of the quantum well of the characteristic size $r_{0}$, which then spreads rapidly to the entire sample. The size of the critical nucleus of the stable current state was found to be bias dependent, $r_{0} \propto\left(V_{t h}-V\right)^{-1 / 4}$. It has been shown that in the large-sample regime $\ln \tau \propto\left(V_{t h}-V\right)$.

These results were obtained for samples whose two lateral dimensions are comparable to each other. In this paper we study the interesting case of the devices of strip geometry with $w \ll L$, where $w$ is the width and $L$ is the length of the strip. In these devices there is a special regime where the applied bias is such that $w \ll r_{0} \ll L$. Since the scale $r_{0}$ gives the characteristic size of the density fluctuations, in this case the density does not vary

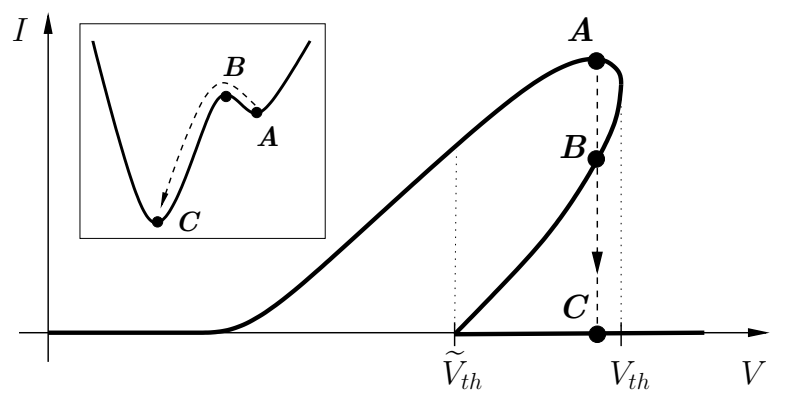

FIG. 1: Current-voltage characteristic of the DBRTS. The bistable region is present in the range of bias between $\widetilde{V}_{t h}$ and $V_{t h}$. The inset shows a sketch of generic potential $u(n)$ in the bistable region. The points $\boldsymbol{A}$ and $\boldsymbol{C}$ on the $I-V$ curve correspond to the local and global minima of $u(n)$, respectively. The point $\boldsymbol{B}$ on the unstable current branch corresponds to the maximum of $u$. 
across the strip, but only along it. Confining the switching process to one dimension alters its properties significantly. Similarly to the two-dimensional case, the switching time grows exponentially when bias is tuned inside the bistable region. However, the exponent follows a different dependence which is found in Sec. III]

We show that in long strips the nucleation can occur either inside or at the ends of the device. It turns out that both nucleation regimes can be observed, but the respective decay times are dramatically different. To compare them, one needs to calculate the switching times with the prefactors. Unlike the case of two-dimensional structures, in one dimension the prefactors can be found exactly in the limit of long and narrow strip, Sec. IV]

The decay of the metastable current state is governed by shot noise, and therefore switching is a stochastic process. We describe this process using the Fokker-Planck equation approach. In Sec.IVA we introduce the FokkerPlanck equation for tunneling and in-plane diffusion in DBRTS. This equation is used to calculate the prefactor of the mean switching time in strip-shaped (Secs. IVB and IVC) and ring-shaped devices (Sec. IVD). We summarize our results and discuss their experimental implications in Sec. D

\section{STATIONARY DISTRIBUTION FUNCTION OF ELECTRON DENSITY}

It was shown in Ref. 12 that the stationary distribution function of electron density $n(\mathbf{r})$ in the quantum well takes the form

$$
P_{0}\{n\}=e^{-F\{n\}}, \quad F\{n\}=\int d^{2} \mathbf{r}\left[u(n)+\eta(\nabla n)^{2}\right] .
$$

Here the integration is over the cross-section of the well. In the case of uniform density $F\{n\}$ is determined by the effective potential $u(n)$, which describes the tunneling between the well and the leads. The gradient term in the functional $F\{n\}$ accounts for the diffusion in the quantum well. The constant $\eta$ is positive and proportional to the in-plane conductivity,${ }^{12} \eta \propto \sigma$. By suppressing gradients of $n$, this term favors the states with uniform density.

The shape of the effective potential $u(n)$ in the bistable region is schematically shown in the inset of Fig. 11 It has the local and global minima at points $\boldsymbol{A}$ and $\boldsymbol{C}$, respectively. In the case of uniform density $n$, these minima result in peaks of the distribution function $P_{0}\{n\}$, which correspond to the upper and lower branches of the $I-V$ curve. Point $\boldsymbol{B}$ on the unstable current branch corresponds to a maximum of $u$.

In the vicinity of the threshold voltage $V_{t h}$ the effective potential $u(n)$ can be approximated by a cubic polynomial

$$
u(n)=-\alpha\left(n-n_{t h}\right)+\frac{\gamma}{3}\left(n-n_{t h}\right)^{3}, \quad \alpha=a\left(V_{t h}-V\right) .
$$

Here $\gamma$ and $a$ are positive constants. The voltage dependence of $\alpha$ ensures that the local minimum of $u$, which corresponds to the metastable state, disappears at the bistability threshold. The threshold density $n_{t h}$ is defined as the density at point $V=V_{t h}$, where the local minimum $\boldsymbol{A}$ of $u(n)$ disappears by merging with the maximum $\boldsymbol{B}$.

If the system is in the local minimum of $u(n)$, it will eventually decay to the global minimum. In the limit of large conductivity $\sigma$ or small sample size the electron density $n$ in the quantum well is uniform, and the gradient term in $F\{n\}$, Eq. (II), can be omitted. In this case Eq. (11) is simplified: the distribution function $P_{0}$ is described by the only variable $n$ and takes the form $P_{0}(n)=e^{-S u(n)}$, where $S$ is the area of the sample. Initially, the system is in the local minimum of $u(n)$, see point $\boldsymbol{A}$ in the inset of Fig. 10 In order to switch to the global minimum the system has to pass through point $\boldsymbol{B}$. As it follows from the expression for $P_{0}(n)$, the probability of reaching point $\boldsymbol{B}$ is exponentially small, with the exponent determined by the barrier height $S\left(u_{B}-u_{A}\right)$. The latter can be easily found from the expansion (2), and the mean switching time $\tau_{0}$ takes the form ${ }^{12}$

$$
\tau_{0}=\tau_{0}^{*} \exp \left(\frac{4}{3} \frac{L w \alpha^{3 / 2}}{\gamma^{1 / 2}}\right) .
$$

Here $\tau_{0}^{*}$ is a preexponential factor, and we assumed that the cross-section of the sample has rectangular shape with the width $w$ and the length $L$.

Expression (33) is valid as long as $L, w \ll r_{0}$, where

$$
r_{0}=\left(\frac{\eta^{2}}{\alpha \gamma}\right)^{1 / 4} \propto\left(V_{t h}-V\right)^{-1 / 4}
$$

has the meaning of the characteristic spatial scale of stochastic fluctuations of electron density $\frac{12}{12}$ The scale $r_{0}$ can be tuned by changing the bias $V$. If $L, w \gg r_{0}$ the switching occurs according to the nucleation scenario. In this case the critical switching density is first achieved in a small part of the sample of size $\sim r_{0}$. After stochastic creation of the critical nucleus, it grows rapidly in size until it occupies the entire sample.

In this paper we consider the case of a very narrow strip, $w \ll L$. In the regime when the bias is such that $w \ll r_{0}$, the density may change only along the strip, and the problem becomes one-dimensional 27

In the following it will be convenient to express the density $n(x)$ in terms of a dimensionless function $z(\xi)$ that vanishes at the minimum of $u(n)$, namely,

$$
n(x)=n_{\min }-2 \sqrt{\frac{\alpha}{\gamma}} z\left(x / r_{0}\right) .
$$

Here the density at the minimum $n_{\min }=n_{t h}+\sqrt{\alpha / \gamma}$. Substituting Eq. (5) into the functional $F$ in Eq. (11), we find

$$
F=U_{1} \int d \xi\left(\frac{z^{\prime 2}}{2}+\frac{z^{2}}{2}-\frac{z^{3}}{3}\right)
$$


where prime denotes differentiation with respect to the dimensionless coordinate $\xi=x / r_{0}$ along the strip. The characteristic value of the functional $F$ is given by the parameter

$$
U_{1}=\frac{8 w \sqrt{\eta} \alpha^{5 / 4}}{\gamma^{3 / 4}}
$$

Its value depends on bias as $U_{1} \propto\left(V_{t h}-V\right)^{5 / 4}$.

\section{THE EXPONENT OF THE MEAN SWITCHING TIME IN LONG STRIPS}

In this section we consider the regime $r_{0} \lesssim L$. In this case, the density fluctuations along the strip result in a significant change of the mean switching time, and the result (3) is no longer applicable. The one-dimensional nature of this problem allows us to obtain an explicit expression for the exponent of the mean switching time $\tau$.

We begin by finding the minimum and the saddle points of the functional $F$ in Eq. (6). They can be found from the condition $\delta F / \delta z=0$, that is,

$$
-\frac{d^{2} z}{d \xi^{2}}+z-z^{2}=0
$$

This equation should be solved with the boundary conditions $z^{\prime}(0)=z^{\prime}(\Lambda)=0$ which account for the fact that there is no current flowing through the ends of the strip. Here we have introduced the dimensionless length of the strip $\Lambda=L / r_{0}$.

Equation (8) can be interpreted as the equation of motion of a classical particle with unit mass in the potential $u(z)=-z^{2} / 2+z^{3} / 3$, see the inset of Fig. 2(a). In this analogy the coordinate $\xi$ plays the role of time. There are two obvious solutions, $z(\xi)=0$ and $z(\xi)=1$, corresponding to the particle staying at the maximum and minimum of $u(z)$, respectively. The minimum of $F\{z\}$ is obviously given by $z(\xi)=0$, since we defined $z$ in such a way that $z=0$ at the minimum. The other solution, $z(\xi)=1$, is a saddle point of $F\{z\}$.

Apart from the two trivial solutions, equation (8) may have $\xi$-dependent solutions corresponding to a moving particle. The boundary conditions $z^{\prime}(0)=z^{\prime}(\Lambda)=0$ require zero velocity at the moments $\xi=0$ and $\xi=\Lambda$. Thus the particle performs oscillatory motion between turning points $z(0)$ and $z(\Lambda)$. If the particle starts at $z(0)=c_{0}$ in the range $0 \leq c_{0} \leq 3 / 2$, one can easily find the other turning point $c$ from the condition $u(c)=u\left(c_{0}\right)$. This equation has two solutions:

$$
c_{ \pm}=\frac{3-2 c_{0} \pm \sqrt{-12 c_{0}^{2}+12 c_{0}+9}}{4} .
$$

The turning point $z(\Lambda)$ corresponds to the positive root $c_{+}$.

The time $\Lambda$ required for the particle to travel from one turning point to the other is obviously one half of the
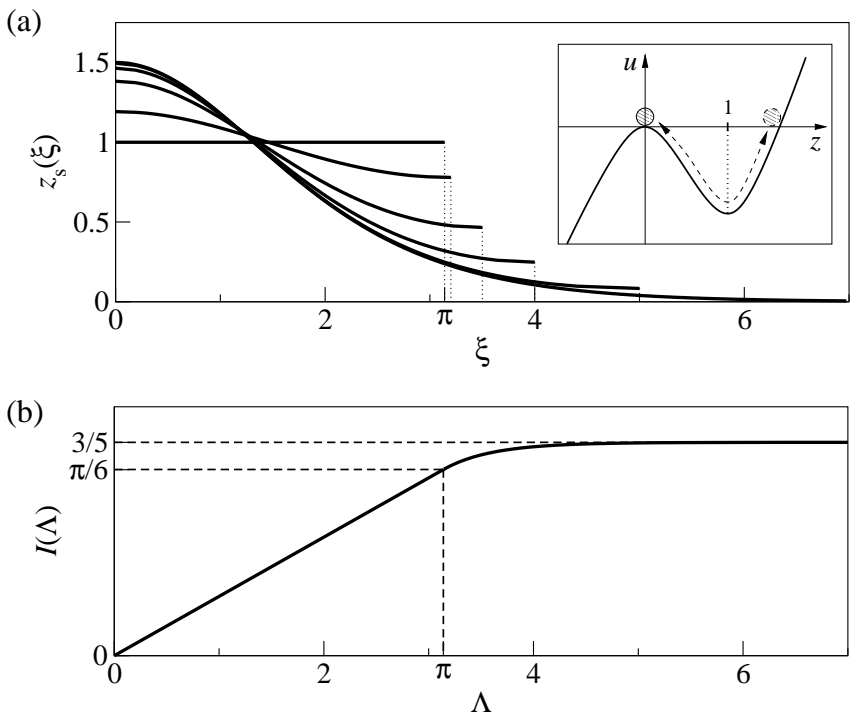

FIG. 2: (a) Saddle point solution $z_{s}(\xi)$ for strips of length $\Lambda=\pi, 3.2,3.5,4,5$, and $\infty$. The allowed motion of a classical particle in the potential $u(z)=-z^{2} / 2+z^{3} / 3$ is shown in the inset. (b) The dependence $I(\Lambda)$ defined by Eq. (13). At $\Lambda<\pi$ it is linear: $I(\Lambda)=\Lambda / 6$; while for $\Lambda>\pi$ it is described by Eq. (14). At $\Lambda \rightarrow \infty$ the saddle point $z_{s}(\xi)$ is given by Eq. (11), and $I=3 / 5$.

period of oscillations. The period of small amplitude oscillations when the particle starts close to the minimum of the potential $u(z)$, i.e. at $c_{0} \rightarrow 1$, equals $2 \pi$. The period monotonically grows to infinity as $c_{0} \rightarrow 3 / 2$ or 0 . Therefore, if $\Lambda<\pi$ there are no $\xi$-dependent solutions, and $z_{s}(\xi)=1$. At $\Lambda>\pi$ we have an additional saddle point $z_{s}(\xi)$ corresponding to a particle moving from $z_{s}(0)=c_{0}$ to $z_{s}(\Lambda)=c_{+}$.

Equation (8) can be solved analytically in terms of the elliptic integrals. In particular, the inverse function of $z_{s}(\xi)$ has the form

$$
\xi\left(z_{s}\right)=\frac{\sqrt{6}}{\sqrt{c_{0}-c_{-}}} F\left(\arcsin \sqrt{\frac{c_{0}-z_{s}}{c_{0}-c_{+}}}, \sqrt{\frac{c_{0}-c_{+}}{c_{0}-c_{-}}}\right),
$$

where $F(\varphi, k)$ is the elliptic integral of the first kind 28 Using Eq. (9) the length of the strip $\Lambda$ can be expressed in terms of $c_{0}$ as

$$
\Lambda=\frac{\sqrt{6}}{\sqrt{c_{0}-c_{-}}} F\left(\frac{\pi}{2}, \sqrt{\frac{c_{0}-c_{+}}{c_{0}-c_{-}}}\right)
$$

Solving equation (10) with respect to $c_{0}$ one can obtain the dependence $c_{0}(\Lambda)$. Substituting it into Eq. (9) and inverting $\xi\left(z_{s}\right)$ one obtains the saddle point $z_{s}(\xi)$ for a given $\Lambda$. The saddle-point solutions $z_{s}(\xi)$ for several values of $\Lambda$ are shown in Fig. 2(a).

For a strip of infinite length the boundary conditions take the form $c_{0}=z_{s}(0)=3 / 2$ and $c_{+}=z_{s}(\infty)=0$. Then expression (9) can be significantly simplified and 
yields

$$
z_{s}(\xi)=\frac{3}{2 \cosh ^{2}(\xi / 2)}
$$

This solution can be easily verified by substitution into Eq. (8).

The mean switching time $\tau$ is given by

$$
\tau=\tau^{*} e^{F\left\{z_{s}\right\}}
$$

where $\tau^{*}$ is a preexponential factor. Using the expression for $F\{z\}$, Eq. (6), we find

$$
F\left\{z_{s}\right\}=U_{1} I(\Lambda), \quad I(\Lambda)=\int_{0}^{\Lambda} d \xi\left(\frac{z^{\prime 2}}{2}+\frac{z_{s}^{2}}{2}-\frac{z_{s}^{3}}{3}\right),
$$

At $\Lambda<\pi$, the only saddle point is $z_{s}=1$, and therefore $I(\Lambda)=\Lambda / 6$. At $\Lambda>\pi$ the saddle point solution $z_{s}(\xi)$ given by Eq. (9) corresponds to a smaller value of $I$. In particular, at $\Lambda \rightarrow \infty$ the solution (11) gives $I=$ $3 / 5$. In the intermediate region the integral $I(\Lambda)$ can be evaluated analytically,

$$
\begin{aligned}
I= & \frac{\Lambda}{60}\left[4 c_{+}^{3}-6\left(c_{+}^{2}+c_{+}+c_{0}-c_{-}\right)+9\right] \\
& +\frac{\sqrt{6}}{5} \sqrt{c_{0}-c_{-}} E\left(\sqrt{\frac{c_{0}-c_{+}}{c_{0}-c_{-}}}\right) .
\end{aligned}
$$

Here $E(k)$ is the complete elliptic integral of the second kind. ${ }^{28}$ The dependence $I(\Lambda)$ is plotted in Fig. 2 (b).

Note that apart from the saddle point described by Eq. (9), for $\Lambda>2 \pi$ there are additional $\xi$-dependent saddle points. For example, there is a $\xi$-dependent solution corresponding to a particle moving from $z_{s}(0)=c_{0}$ to $z_{s}(\Lambda)=c_{+}$and back to $c_{0}$. This saddle point is responsible for the processes of switching inside the strip, see Sec.IVC At $\Lambda>3 \pi$ another saddle point appears which corresponds to a particle moving from $c_{0}$ to $c_{+}$, returning to $c_{0}$, and back to $c_{+}$. In general, for $\Lambda$ between $\pi m$ and $\pi(m+1)$ there exist $m$ different $\xi$-dependent solutions. However, the additional saddle points give larger values of $F\left\{z_{s}\right\}$ and thus do not affect the switching time.

\section{PREFACTOR OF THE SWITCHING TIME}

The exponential dependence of the mean switching time (12) was obtained from the stationary distribution function $P_{0}\{n\}$ of electron density. However, to calculate the prefactor $\tau^{*}$, understanding of the time evolution of the distribution function $P\{n(x), t\}$ is also required. When electrons tunnel in or out of the well, the density $n(x)$ changes in very small increments. Thus the dynamics of $P\{n(x), t\}$ is described by a Fokker-Planck equation. ${ }^{12}$ If the strip is very short, the density in the quantum well is uniform, and the system dynamics is described by a single variable $n$. In this case the FokkerPlanck equation for $P(n, t)$ essentially coincides with the one for a small two-dimensional sample. Then by using conventional techniques ${ }^{29}$ the prefactor of the switching time can be found 12 as

$$
\tau_{0}^{*}=\frac{2 \pi}{b \sqrt{\alpha \gamma}}
$$

This result is correct as long as $\Lambda \ll 1$.

In longer strips the density fluctuates, and therefore the Fokker-Planck equation with one variable $n$ cannot adequately describe the evolution of the distribution function $P$. In this case $P$ is a functional of $n(x)$, and the Fokker-Planck equation is multidimensional. Then to study the decay of metastable current states one should use a more sophisticated method.

\section{A. Fokker-Planck approach to current switching in DBRTS}

To find the prefactor of the mean switching time for the system described by the multidimensional Fokker-Planck equation, we use the first passage time technique ${ }^{30.31}$ This method is applied to the Fokker-Planck equation in the form

$$
\begin{aligned}
& \frac{\partial P(\mathbf{x}, t)}{\partial t}=\mathcal{L} P(\mathbf{x}, t) \\
& \mathcal{L}=-\sum_{i} \frac{\partial}{\partial x_{i}} K_{i}(\mathbf{x})+\sum_{i, j} \frac{\partial^{2}}{\partial x_{i} \partial x_{j}} D_{i j}(\mathbf{x})
\end{aligned}
$$

where the matrix $D_{i j}$ represents the generic diffusion coefficient, and $\mathbf{K}$ is the drift field. Assuming that the system has a metastable state, one can consider its domain of attraction $\Omega$ with the domain boundary $\partial \Omega$ being the separatrix of the field $\mathbf{K}$. For the stochastic process described by Eq. (16), the mean time of the first passage out of the domain $\Omega$ has been found in Refs. 30.31 . The mean switching time is obtained as doubled mean first-passage time and takes the form, ${ }^{31}$

$$
\tau=-\frac{2 \int_{\Omega} d^{d} x P_{0}(\mathbf{x})}{\sum_{i} \int_{\partial \Omega} d S_{i} \sum_{j} D_{i j}(\mathbf{x}) P_{0}(\mathbf{x}) \frac{\partial f(\mathbf{x})}{\partial x_{j}}} .
$$

Here $P_{0}$ is the stationary solution of Eq. (16). The form function $f(\mathbf{x})$ is defined as a stationary solution of the adjoint equation,

$$
\mathcal{L}^{\dagger} f(\mathbf{x}, t)=\sum_{j}\left(K_{j}(\mathbf{x})+\sum_{i} D_{i j}(\mathbf{x}) \frac{\partial}{\partial x_{i}}\right) \frac{\partial f(\mathbf{x})}{\partial x_{j}}=0 .
$$

It satisfies the boundary conditions $f(\mathbf{x})=1$ inside the domain $\Omega$ and $f(\mathbf{x})=0$ at the boundary $\partial \Omega$.

The Fokker-Planck equation for the case of nonuniform electron density $n(\mathbf{r})$ in the well was obtained in Ref. 12. It takes the form

$$
\frac{\partial P\{n, t\}}{\partial t}=\frac{b}{2} \int d \mathbf{r} \frac{\delta}{\delta n}\left(u^{\prime}(n)-2 \eta \nabla^{2} n+\frac{\delta}{\delta n}\right) P\{n, t\} .
$$


It is easy to check that the stationary solution of this equation is given by Eq. (1).

Close to the threshold $u(n)$ can be approximated by the expansion (2), and the Fokker-Planck equation (19) can be represented in terms of dimensionless variable $z(\xi)$, Eq. (5), as

$\frac{\partial P\{z, \Theta\}}{\partial \Theta}=\int d \xi \frac{\delta}{\delta z}\left(-\frac{d^{2} z}{d \xi^{2}}+z-z^{2}+\frac{1}{U_{1}} \frac{\delta}{\delta z}\right) P\{z, \Theta\}$

where $\Theta=2 \pi t / \tau_{0}^{*}$ is the dimensionless time. The stationary solution $P_{0}(z)$ of this equation is $e^{-F}$ with $F\{z\}$ given by Eq. (6). Equation (20) has an infinite number of variables, since the density $z(\xi)$ is different at every point. From now on we can consider a purely mathematical problem of decay of a metastable state for the system governed by dimensionless equation (20). This equation is rather generic and we expect it to describe other one-dimensional problems of decay of metastable states.

\section{B. Nucleation at an end of a strip}

To find the prefactor of the mean switching time (12) we use expression (17). We evaluate both integrals in Eq. (17) in Gaussian approximation. The integral in the numerator of Eq. (17) is dominated by the minimum of $F\{z\}$. To find the expression for $F$ in the vicinity of its minimum, it is convenient to use Fourier expansion

$$
z(\xi)=\frac{x_{0}}{\sqrt{\Lambda}}+\sum_{i=1}^{\infty} x_{i} \phi_{i}(\xi), \quad \phi_{i}(\xi)=\sqrt{\frac{2}{\Lambda}} \cos \left(\frac{\pi i}{\Lambda} \xi\right) .
$$

Substituting this expansion into Eq. (6), we find that up to quadratic in $x_{i}$ terms,

$$
F(\mathbf{x})=\frac{U_{1}}{2} \sum_{i=0}^{\infty} \lambda_{i} x_{i}^{2}
$$

where we defined $\lambda_{i} \equiv(\pi i / \Lambda)^{2}+1$. Using this expression for $F$, one can easily evaluate the integral in the numerator of Eq. (17),

$$
\int_{-\infty}^{\infty} \prod_{i} d x_{i} e^{-F(\mathbf{x})}=\prod_{i} \sqrt{\frac{2 \pi}{U_{1} \lambda_{i}}}
$$

The integral in the denominator of Eq. (17) is dominated by the saddle point $z_{s}(\xi)$ of the functional $F\{z\}$. To find the expression for $F$ in the vicinity of the saddle point it is convenient to expand $z(\xi)$ near $z_{s}(\xi)$ as

$$
z(\xi)=z_{s}(\xi)+\sum_{i=0}^{\infty} \tilde{x}_{i} \tilde{\phi}_{i}(\xi)
$$

Here $\tilde{\phi}_{i}(\xi)$ are the normalized solutions of the eigenvalue problem

$$
\left(-\frac{d^{2}}{d \xi^{2}}-2 z_{s}(\xi)+1\right) \tilde{\phi}_{i}(\xi)=\tilde{\lambda}_{i} \tilde{\phi}_{i}(\xi)
$$

with the boundary conditions

$$
\tilde{\phi}_{i}^{\prime}(0)=0, \quad \tilde{\phi}_{i}^{\prime}(\Lambda)=0
$$

Substituting Eq. (24) into $F\{z\}$ given by Eq. (6), and expanding near $\tilde{\mathbf{x}}=0$ up to the second order in $\tilde{x}_{i}$, we find

$$
F\{\tilde{\mathbf{x}}\}=U_{1} I(\Lambda)+\frac{U_{1}}{2} \sum_{i=0}^{\infty} \tilde{\lambda}_{i} \tilde{x}_{i}^{2}
$$

where the first term is given by Eq. (13).

Expression (27) implies that it is convenient to calculate the integral in the denominator of Eq. (17) in terms of variables $\tilde{x}_{i}$. Since the expansion coefficients $\tilde{x}_{i}$ are related to $x_{i}$ by orthogonal trasformation, the integrals over $x_{i}$ in the denominator of Eq. (17) can be replaced by those over $\tilde{x}_{i}$.

To calculate the integral in the denominator of Eq. (17) we also need to find $D_{i j}$ and $\partial f / \partial \tilde{x}_{j}$. They can be obtained from the $\tilde{\mathbf{x}}$-representation of the Fokker-Planck equation (20). The adjoint to the operator $\mathcal{L}$ of Eq. (20) in the $\tilde{\mathbf{x}}$-representation can be written as

$$
\mathcal{L}^{\dagger}=\sum_{i=0}^{\infty}\left(-\tilde{\lambda}_{i} \tilde{x}_{i} \frac{\partial}{\partial \tilde{x}_{i}}+\frac{1}{U_{1}} \frac{\partial^{2}}{\partial \tilde{x}_{i}^{2}}\right) .
$$

Here the terms of higher orders in $\tilde{x}_{i}$ were neglected.

In the denominator of Eq. (17) the boundary $\partial \Omega$ is orthogonal to the unstable direction $\tilde{x}_{0}$ on the saddle. Therefore the sum over $i$ reduces to the only term $i=0$. Using the definition of $D_{i j}$ we find from Eq. (28) that $D_{i j}=U_{1}^{-1} \delta_{i j}$. The sum over $j$ then reduces to a single term $j=0$, and we need to find only the derivative $\partial f / \partial \tilde{x}_{0}$, which is given by equation (18) in $\tilde{\mathbf{x}}$ representation. The latter equation takes the form

$$
\left(-\tilde{\lambda}_{0} \tilde{x}_{0}+\frac{1}{U_{1}} \frac{\partial}{\partial \tilde{x}_{0}}\right) \frac{\partial f}{\partial \tilde{x}_{0}}=0 .
$$

Since the saddle point is unstable in the $\tilde{x}_{0}$-direction, the eigenvalue $\tilde{\lambda}_{0}$ is negative. Thus equation (29) can be solved with the boundary conditions $f=1$ at $\tilde{x}_{0} \rightarrow-\infty$ and $f=0$ at the domain boundary $\tilde{x}_{0}=0$ required by the definition of $f(\tilde{\mathbf{x}})$, cf. Eq. (18). As a result, we obtain $\partial f / \partial \tilde{x}_{0}=-\left(2\left|\tilde{\lambda}_{0}\right| U_{1} / \pi\right)^{1 / 2}$ at the saddle point.

Substituting Eqs. (23) and (27) into Eq. (17) we express the switching time in a strip-shaped device $\tau_{s}$ as

$$
\tau_{s}=\tau_{s}^{*} e^{U_{1} I(\Lambda)}, \quad \tau_{s}^{*}=\frac{\tau_{0}^{*}}{\sqrt{\left|\tilde{\lambda}_{0}\right|}} \sqrt{\frac{\tilde{\lambda}_{1}}{\lambda_{1}}} \Upsilon_{s}
$$

where

$$
\Upsilon_{s}=\prod_{i=2}^{\infty} \sqrt{\frac{\tilde{\lambda}_{i}}{\lambda_{i}}}
$$

Thus the evaluation of the prefactor of the switching time reduces to solving the eigenvalue problem (25), (26). 


\section{Very long strip}

We first consider the limit of a very long strip, $\Lambda \gg 1$. Then the saddle point $z_{s}(\xi)$ is given by Eq. (11), so the equation (25) takes the form

$$
\left(-\frac{d^{2}}{d \xi^{2}}-\frac{3}{\cosh ^{2}(\xi / 2)}+1\right) \tilde{\phi}_{i}(\xi)=\tilde{\lambda}_{i} \tilde{\phi}_{i}(\xi),
$$

The eigenvalue problem (32) with boundary conditions (26) is solved analytically in the Appendix . In particular, we find that the discrete spectrum consists of two bound states with eigenvalues $\tilde{\lambda}_{0}=-5 / 4$ and $\tilde{\lambda}_{1}=3 / 4$. At finite $\Lambda$ the continuous spectrum is discretized according to

$$
\tilde{\lambda}_{i}=1+\left(\frac{\pi i}{\Lambda}\right)^{2}\left(1-\frac{\delta(\pi i / \Lambda)}{\pi i}\right)^{2},
$$

where the scattering phase shift $\delta(q)$ has the form

$$
\delta(q)=\sum_{n=1}^{3} \arctan \frac{n}{2 q} .
$$

To evaluate $\Upsilon_{s}$ it is convenient to take the logarithm of Eq. (31), thereby converting the infinite product to a sum. In the limit $\Lambda \rightarrow \infty$, we find

$$
\ln \Upsilon_{s}=-\frac{1}{\pi} \int_{0}^{\infty} \frac{q \delta(q)}{1+q^{2}} d q=\frac{1}{2} \ln \frac{2}{15} .
$$

Using this result for $\Upsilon_{s}$ and the fact that in a long strip $\lambda_{1} \rightarrow 1$, we find the mean time of switching in a very long strip

$$
\tau_{e}=\frac{\sqrt{2}}{5} \tau_{0}^{*} \exp \left(\frac{3 U_{1}}{5}\right) .
$$

It is important to note that the above calculation accounts for only one of two equivalent saddle points in a strip-shaped device. Indeed, the saddle point fluctuation (11) is positioned near the left end of the sample, $\xi=0$. Alternatively, the density fluctuation could occur at the right end of the device. This possibility is formally described by considering the saddle point $z_{s}(\Lambda-\xi)$. Since the two types of processes are equivalent, the total rate of switching at both ends of the device is $2 / \tau_{e}$.

\section{Strip of arbitrary length}

Another regime in which analytical expression for $\tau_{s}$ can be obtained is that of $\Lambda<\pi$. In this case the saddlepoint density is uniform, $z_{s}(\xi)=1$. Substituting it into equation (25) we find $\tilde{\lambda}_{i}=\lambda_{i}-2$. Then expression (30), (31) simplifies significantly, and the prefactor of $\tau_{s}$ becomes

$$
\tau_{s}^{*}=\tau_{0}^{*} \prod_{i=1}^{\infty} \sqrt{\frac{(\pi i)^{2}-\Lambda^{2}}{(\pi i)^{2}+\Lambda^{2}}} .
$$

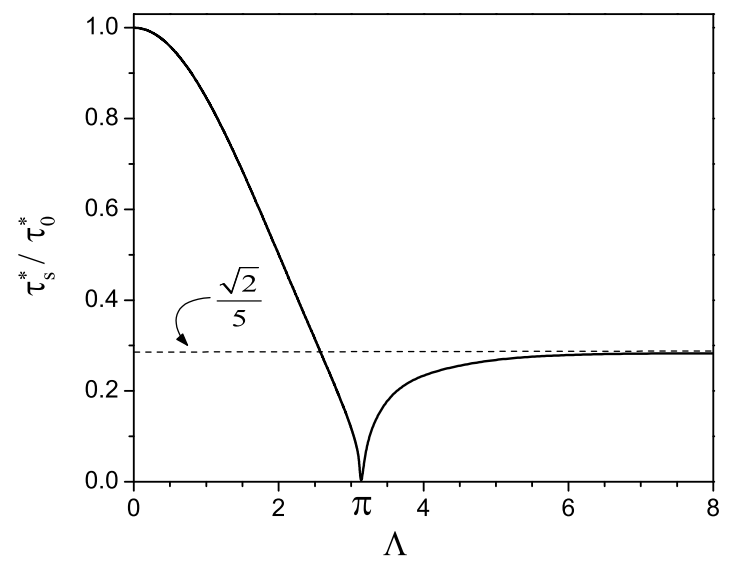

FIG. 3: Prefactor $\tau_{s}^{*}$ of the mean switching time in units $\tau_{0}^{*}$ vs. the dimensionless length of the strip $\Lambda$. At $\Lambda<\pi$ the prefactor is given by Eq. (37), whereas above $\pi$ it is calculated numerically. At $\Lambda \rightarrow \infty$ the prefactor approaches $\sqrt{2} / 5$ in agreement with Eq. (36).

Evaluating the infinite product we obtain

$$
\tau_{s}^{*}=\tau_{0}^{*} \sqrt{\frac{\sin \Lambda}{\sinh \Lambda}} .
$$

This result generalizes the formula (3) for the switching time in short devices, $\Lambda \ll 1$, to the case of any $\Lambda<\pi$.

For a strip of finite length $\Lambda>\pi$, the saddle point density $z_{s}(\xi)$ is given by Eqs. (9) and (10). In this case the eigenvalues $\tilde{\lambda}_{i}$ are obtained by solving numerically the eigenvalue problem (25) with boundary conditions (26) for a given $\Lambda$. Substituting them into expression (30), (31) we find the switching time. The result for the prefactor of $\tau_{s}$ as a function of $\Lambda$ is shown in Fig. 3 .

Let us discuss the behavior of the prefactor at $\Lambda=$ $\pi$. At $\Lambda<\pi$ the functional $F\{z\}$ has only the uniform saddle point $z_{s}(\xi)=1$, whereas at $\Lambda>\pi$ there are two saddle points, the uniform one and the $\xi$-dependent one, see Fig. 2(a). These two saddle points merge at $\Lambda=\pi$. Formally, this gives rise to the fact that $\tilde{\lambda}_{1}$ vanishes at $\Lambda=\pi$, and therefore the prefactor $\tau_{s}^{*}=0$. We believe that the prefactor remains nonzero at $\Lambda=\pi$; however, the evaluation of the prefactor in this case requires a more careful treatment than the Gaussian approximation used in this paper. We leave this problem for future work.

It is worth mentioning that the non-monotonic behavior of the prefactor, see Fig. 3, does not result in nonmonotonic dependence of the switching time on sample length $\Lambda$. The reason is that the prefactor $\tau_{s}^{*}$ is multiplied by the very large exponential [see Eqs. (12)-(13) and Fig. [2(b)] which monotonically grows with $\Lambda$. Therefore, the expression (12) with the prefactor $\tau_{s}^{*}$ and exponent (13) is monotonic everywhere except for the very narrow region $\pi-3 / U_{1} \lesssim \Lambda<\pi$. As discussed in the 
previous paragraph, a more accurate evaluation of the prefactor is required to evaluate the prefactor near $\Lambda=\pi$, and we expect that it would restore the monotonicity of $\tau$ as the function of $\Lambda$.

\section{Nucleation inside long strips and in rings of large circumference}

Apart from the ends of a strip, nucleation can occur inside the sample. Such processes are most important in ring-shaped samples, which have no ends. Thus to study interior switching we model the sample by a strip with periodic boundary conditions. In the following it will be convenient to consider a strip of length $2 \Lambda$ with boundary conditions $z(-\Lambda)=z(\Lambda), z^{\prime}(-\Lambda)=z^{\prime}(\Lambda)$.

To obtain the switching time we first need to find the saddle point of $F\{z\}$. In the mechanical analogy used in Sec. III [see also the inset of Fig. 2(a)] the periodic boundary conditions for the saddle-point equation correspond to a full period of oscillations of the particle, rather than half period as in the case of nucleation at the ends in Sec. IVB. One such solution is given by $z_{s}(|\xi|)$ on the interval $-\Lambda<\xi<\Lambda$, where $z_{s}(\xi)$ is defined by Eqs. (9) and (10), see also Fig. 2(a). [Additional solutions are obtained by shifts $z_{s}(\xi) \rightarrow z_{s}(\xi+\Delta \xi)$.] To find the exponent of the switching time, we need to calculate $F\left\{z_{s}\right\}$ using Eq. (6). Since the saddle-point solution $z_{s}(|\xi|)$ is a symmetric function on the interval $-\Lambda<\xi<\Lambda$, the integral in $F\left\{z_{s}\right\}$ is doubled compared to that of end switching. Thus the time of the switching inside the strip takes the form

$$
\tau_{r}=\tau_{r}^{*} e^{2 I(\Lambda) U_{1}}
$$

\section{cf. Eq. (30).}

The calculation of the prefactor $\tau_{r}^{*}$ is similar to the one for the switching at an end of the strip (Sec. IVB). As in Sec. IVB it is convenient to evaluate the denominator of Eq. (17) by expanding $z(\xi)$, Eq. (24), where $\tilde{\phi}_{i}(\xi)$ are again the eigenfunctions of equation (32), but now with periodic boundary conditions

$$
\tilde{\phi}_{i}(-\Lambda)=\tilde{\phi}_{i}(\Lambda), \quad \tilde{\phi}_{i}^{\prime}(-\Lambda)=\tilde{\phi}_{i}^{\prime}(\Lambda) .
$$

The eigenvalue problem (32), (39) is solved in the Appendix . The discrete spectrum consists of one negative eigenvalue $\tilde{\lambda}_{0}=-5 / 4$, one zero eigenvalue $\tilde{\lambda}_{1}=0$, and $\tilde{\lambda}_{2}=3 / 4$; all other eigenvalues are positive and belong to the quasicontinuous spectrum, $\tilde{\lambda}_{i} \geq 1$ for $i>2$. In the denominator of Eq. (17) the integration over the amplitudes $\tilde{x}_{2}, \tilde{x}_{3}, \tilde{x}_{4}, \ldots$ of the modes with positive eigenvalues is easily performed in Gaussian approximation. The integration over the amplitude $\tilde{x}_{1}$ of the zero mode is less trivial.

Let us discuss the physical origin of the zero mode. The functional $F\{z\}$ is translationally invariant on a ring, and therefore $F\left\{z_{s}\right\}$ does not change if the saddle-point solution is shifted, $z_{s}(\xi) \rightarrow z_{s}(\xi+\Delta \xi)$. In other words, the deformation $\delta z_{s}=\Delta \xi z_{s}^{\prime}(\xi)$ does not affect $F$. On the other hand, according to Eqs. (24) and (27) the deformation $\delta z_{s}=\tilde{x}_{1} \tilde{\phi}_{1}(\xi)$ of the saddle-point solution does not change $F$ either, because $\tilde{\lambda}_{1}=0$. Since the zero mode is unique, we conclude

$$
\Delta \xi z_{s}^{\prime}(\xi)=\tilde{x}_{1} \tilde{\phi}_{1}(\xi)
$$

Then it follows that ${ }^{32} z_{s}^{\prime}(\xi)=c_{1} \tilde{\phi}_{1}(\xi)$. The constant $c_{1}$ can be found from the normalization condition for the eigenfunctions $\tilde{\phi}_{i}(\xi)$,

$$
c_{1}=\sqrt{\int\left[z_{s}^{\prime}(\xi)\right]^{2} d \xi} .
$$

Using Eq. (40) and the relation $z_{s}^{\prime}(\xi)=c_{1} \tilde{\phi}_{1}(\xi)$, the integral over the amplitude $\tilde{x}_{1}$ of the zero mode takes the form

$$
\int d \tilde{x}_{1}=c_{1} \int d(\Delta \xi)=2 c_{1} \Lambda .
$$

Expressions for $\partial f / \partial \tilde{x}_{0}$ and $D_{i j}$ were obtained in Sec. IV] independently of the exact form of the saddlepoint density and are therefore still applicable. Substituting expression (23) for the numerator of Eq. (17) and using Eq. (42) in the denominator, we find

$$
\tau_{r}^{*}=\frac{\sqrt{\pi} \tau_{0}^{*}}{\sqrt{2\left|\tilde{\lambda}_{0}\right| \lambda_{1}} c_{1} \Lambda \sqrt{U_{1}}} \sqrt{\frac{\tilde{\lambda}_{2}}{\lambda_{2}}} \Upsilon_{r} .
$$

Here the infinite product $\Upsilon_{r}$ is similar to $\Upsilon_{s}$ evaluated in Sec. IVB Eq. (31), but with the eigenvalues $\lambda_{i}$ and $\tilde{\lambda}_{i}$ calculated with periodic boundary conditions (39). It is shown in the Appendix that at $\Lambda \gg 1$ the quasicontinuous spectrum of the eigenvalue problem (32), (39) is still given by Eq. (33), but becomes doubly degenerate. Therefore, in the limit of an infinite strip the product $\Upsilon_{r}$ can be found as

$$
\Upsilon_{r}=\Upsilon_{s}^{2}=\frac{2}{15}
$$

cf. Eq. (35). Also, substituting expression (11) for $z_{s}(\xi)$ into Eq. (41) we find $c_{1}=\sqrt{6 / 5}$ at $\Lambda \gg 1$. Upon substitution of these results into Eq. (43) the mean time of switching inside a long strip takes the form,

$$
\tau_{i}=\frac{\sqrt{\pi}}{15 \Lambda} \frac{\tau_{0}^{*}}{\sqrt{U_{1}}} \exp \left(\frac{6 U_{1}}{5}\right) .
$$

where $U_{1}$ and $\tau_{0}^{*}$ are given respectively by Eqs. (7) and (15), and $\Lambda=L / 2 r_{0}$. To obtain the exponent we used the fact that the integral $I=3 / 5$ at $\Lambda \gg 1$.

In the case of a device of strip geometry, switching can be initiated both at the ends and inside the sample. The exponent of $\tau_{i}$ is a factor of 2 larger than the exponent of $\tau_{e}$ [see Eq. (36)], which makes the switching at the ends generally more favorable. On the other hand, the rate $1 / \tau_{i}$ is proportional to the strip length $L$. Therefore in very long strips interior switching denominates. 


\section{Nucleation in rings of arbitrary circumference}

Expression (45) was obtained in the limit $L \gg r_{0}$. In this section we discuss the case of a ring of finite circumference $L \lesssim r_{0}$. We will consider separately the cases $L<2 \pi r_{0}$ and $L>2 \pi r_{0}$. We will use the expression (38) for the exponential of the switching time, which was obtained for an arbitrary circumference of the ring.

Let us start with the case $L<2 \pi r_{0}$. In dimensionless units it corresponds to the problem of a ring with the circumference $2 \Lambda<2 \pi$. Similarly to the case of a strip of length $\Lambda<\pi$, see Sec.IVB2 the saddle-point density is uniform, $z_{s}(\xi)=1$, and from equation (25) we obtain $\tilde{\lambda}_{i}=\lambda_{i}-2$. Analogously to the result (30), (31) for the strip geometry the prefactor takes the form

$$
\tau_{r}^{*}=\frac{\tau_{0}^{*}}{\sqrt{\left|\tilde{\lambda}_{0}\right|}} \prod_{i=1}^{\infty} \sqrt{\frac{\tilde{\lambda}_{i}}{\lambda_{i}}}
$$

Since the boundary conditions for the ring are periodic, Eq. 25 has two types of solutions, $\cos (\pi i \xi / \Lambda)$ and $\sin (\pi i \xi / \Lambda)$. The two solutions have the same eigenvalues for any $i>0$, and thus all egenvalues except for $\tilde{\lambda}_{0}$ are doubly degenerate. Repeating calculations similar to those for Eq. (37), we find

$$
\tau_{i}^{*}=\tau_{0}^{*} \frac{\sin \Lambda}{\sinh \Lambda} .
$$

This expression is similar to the result (37) for the strip of length $\Lambda<\pi$. The absence of the square root in the righthand side of Eq. (47) is due to the double degeneracy of the eigenvalues.

At $\Lambda>\pi$ one needs to solve the eigenvalue problem (25) with nonuniform saddle point $z_{s}(\xi)$, Eqs. (9) and (10), which can be done numerically. Then one substitutes the eigenvalues $\tilde{\lambda}_{i}$ into Eq. (43) to find the prefactor $\tau_{r}^{*}$. As we found in the Appendix, in the limit $\Lambda \rightarrow \infty$ the three lowest eigenvalues $\tilde{\lambda}_{i}$ are nondegenerate whereas the rest of them are doubly degenerate, because the potential $-2 z_{s}(\xi)$ is then reflectionless. Unexpectedly, our numerical calculation shows that the same property holds for any $\Lambda>\pi$. Then similar to Eq. (44) we find $\Upsilon_{r}=\Upsilon_{s}^{2}$, where $\Upsilon_{s}$ defined by Eq. (31) was computed in Sec. IVB2 The prefactor $\tau_{i}^{*}$ above $\Lambda=\pi$ is plotted as a function of $\Lambda$ in Fig. 4. One can see in Fig. 4 that at $\Lambda \rightarrow \infty$ the prefactor approaches 0 as $1 / \Lambda$ in agreement with Eq. (45).

Contrary to the case of a strip, see Fig. 3 the prefactor $\tau_{r}^{*}$ does not vanish as $\Lambda$ approaches $\pi$ from above. This is due to the fact that at $\Lambda \rightarrow \pi$ small $\tilde{\lambda}_{2}$ in the numerator of Eq. (43) is compensated by small $c_{1}$ in the denominator. To see this let us consider the saddle point solution close to $\Lambda=\pi$. At $\Lambda=\pi+\epsilon$ with $\epsilon \ll 1$ this saddle point can be expanded up to its second harmonic as

$$
z_{s}=1+\kappa_{0}+\kappa_{1} \cos (\pi \xi / \Lambda)+\kappa_{2} \cos (2 \pi \xi / \Lambda)
$$

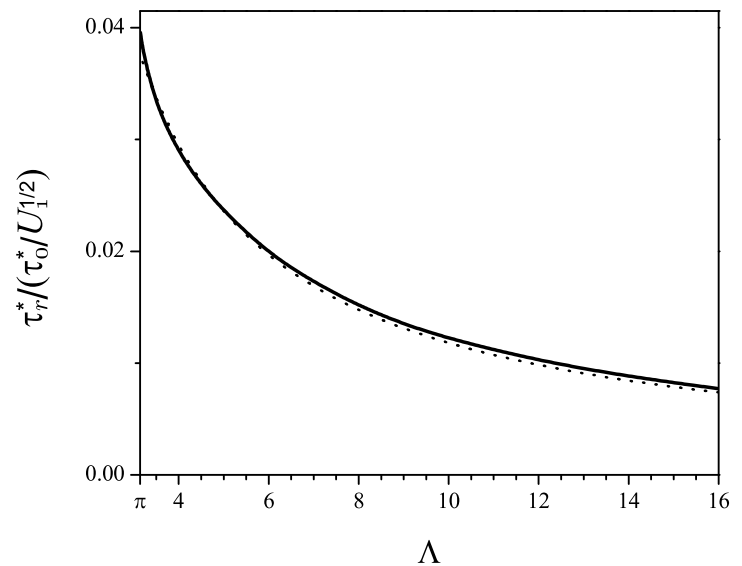

FIG. 4: Prefactor $\tau_{r}^{*}$ of the mean switching time in units $\tau_{0}^{*} / \sqrt{U_{1}}$ vs. the dimensionless circumference of the ring $2 \Lambda$ above $\Lambda=\pi$. The dotted line shows the asymptote $\sqrt{\pi} / 15 \Lambda$ of $\tau_{r}^{*}$ at $\Lambda \rightarrow \infty$, see Eq. (45).

where $\kappa_{0}=-\kappa_{1}^{2} / 2, \kappa_{2}=\kappa_{1}^{2} / 6$, and $\epsilon=5 \pi \kappa_{1}^{2} / 12$. Then from Eq. (41) we obtain $c_{1}=\sqrt{\pi} \kappa_{1}$, and calculating $\tilde{\lambda}_{2}$ up to second order in $\kappa_{1}$ we find $\tilde{\lambda}_{2}=5 \kappa_{1}^{2} / 3$. As a result the ratio $\sqrt{\tilde{\lambda}_{2}} / c_{1}$ in Eq. (43) remains finite, and the prefactor at $\Lambda \rightarrow \pi^{+}$takes the form

$$
\tau_{r}^{*}=\frac{\sqrt{5}}{2 \sqrt{6} \sinh \pi} \frac{\tau_{0}^{*}}{\sqrt{U_{1}}} .
$$

Similar to the case of a finite strip, the singular behavior of $\tau_{r}^{*}$ near $\Lambda=\pi$ is an artifact of Gaussian approximation. We expect that a more careful treatment will show that the prefactor remains nonzero and continuous at $\Lambda=\pi$.

\section{DISCUSSION}

We have studied the mean time $\tau$ of switching from the metastable to the stable state in one-dimensional double-barrier resonant tunneling structures. We calculated both the exponentials and prefactors of $\tau$ for the strip and ring geometries of the sample. In this section we discuss the behavior of the mean switching time in a strip-shaped sample depending on the distance from the threshold and the structural parameters of DBRTS.

In the very vicinity of the threshold the exponent of the mean switching time is of order unity and the metastable state decays very rapidly. As the voltage $V$ is tuned further inside the bistable region, the decay time becomes exponentially long. Our results are applicable in this regime. Thus the exponents in the expressions for the switching time in the regime of short strip, Eq. (3), and in the regime of long strip, Eqs. (36) and (45), must be much 
(a)

(b)
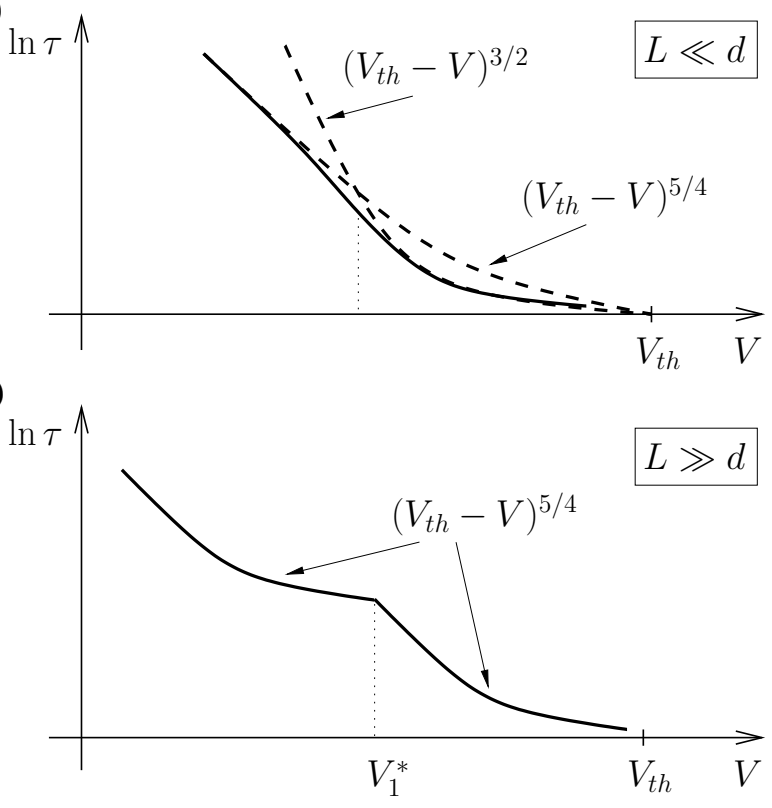

FIG. 5: (a) Logarithm of the mean switching time $\tau$ vs. voltage at $L \ll d$. Near the threshold there is a region of $3 / 2$ power law dependence of $\ln \tau$ (regime of short strip), then follows the region of 5/4-dependence corresponding to the switching at the ends in the regime of long strip. (b) Logarithm of the switching time $\tau$ vs. voltage at $L \gg d$. Close to the threshold one first observes 5/4-power law behavior corresponding to the interior switching, then at voltage below $V_{1}^{*}$ follows the region of 5/4-dependence corresponding to switching at the ends.

greater than unity. To check whether these conditions are satisfied, it is convenient to introduce a bias-independent characteristic length

$$
d \equiv r_{0}\left(\frac{3 U_{1}}{5}\right)^{1 / 5}=\left(\frac{24}{5} \frac{w \eta^{3}}{\gamma^{2}}\right)^{1 / 5}
$$

chosen in such a way that the exponent in Eq. (36) takes the simple form $\left(d / r_{0}\right)^{5}$.

The exponent of the mean switching time in the shortstrip regime [Eq. (3)] can be expressed in terms of $d$ and $r_{0}$ as

$$
\frac{4 L w \alpha^{3 / 2}}{3 \gamma^{1 / 2}}=\frac{5}{18} \frac{L}{r_{0}}\left(\frac{d}{r_{0}}\right)^{5}
$$

This exponent is much greater than unity at $r_{0} \ll$ $\left(L d^{5}\right)^{1 / 6}$. Since the regime of short strip is defined by $r_{0} \gg L$, it is present only if $L \ll d$. In this case, starting at voltage difference $V_{t h}-V$ corresponding to $r_{0} \sim\left(L d^{5}\right)^{1 / 6}$, there is a region of $3 / 2$-power law dependence of $\ln \tau$ corresponding to the short-strip regime. Then, as $V_{t h}-V$ reaches the value corresponding to $r_{0} \sim L$, follows the region of 5/4-dependence of $\ln \tau$ for the long-strip regime, see Fig. 1 (a).

In the latter case the switching can be initiated either inside or at each of the two ends of the sample. Summing the rates of these processes we find

$$
\frac{1}{\tau}=\frac{2}{\tau_{e}}+\frac{1}{\tau_{i}} .
$$

To determine the type of $5 / 4$-dependence that can be observed in the regime of long strip, $r_{0} \ll L$, one should compare the rates of switching inside and at the ends of the strip. Using Eqs. (36) and (45), the ratio of the rates $\tau_{e}^{-1}$ and $\tau_{i}^{-1}$ can be expressed as

$$
\frac{\tau_{e}^{-1}}{\tau_{i}^{-1}} \sim \frac{d}{L} \exp \left[\left(\frac{d}{r_{0}}\right)^{5}-\frac{7}{2} \ln \frac{d}{r_{0}}\right] .
$$

Using this expression we conclude that at $L \ll d$ the switching always initiates at the ends rather than inside the strip. Thus the region of 5/4-dependence is described by Eq. (36).

At $L \gg d$ there is no region corresponding to the regime of a short strip. One can only observe 5/4-power law dependence of $\ln \tau$ corresponding to the long-strip regime. Analyzing Eq. (53) one can see that at $L \gg d$ there are two distinct regions of 5/4-power law dependences. At $r_{0} \lesssim d$ and very large $L$ the switching initiates inside the strip. At very small $r_{0}$ the exponential in Eq. (53) becomes very large, and therefore the switching takes place at the ends. The voltage $V_{1}^{*}$, at which the crossover between these two regions occurs, is given by the condition $\tau_{i}^{-1}=\tau_{e}^{-1}$ applied to Eq. (53),

$$
V_{1}^{*} \approx V_{t h}-\frac{\eta^{2}}{\gamma a d^{4}}\left(\ln \frac{L}{d}\right)^{4 / 5} .
$$

Thus we conclude that in the case of $L \gg d$, starting at $V$ corresponding to $r_{0} \sim d$, one first observes 5/4-power law dependence of $\ln \tau$ on voltage corresponding to the switching inside the strip, then at $V$ below $V_{1}^{*}$ follows the region of 5/4-dependence corresponding to the switching

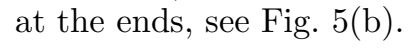

\section{Acknowledgments}

The authors are grateful to S. W. Teitsworth and M. B. Voloshin for fruitful discussions. O.A.T. acknowledges the hospitality of Argonne National Laboratory where part of this work was performed. This work was supported by NSF Grant No. DMR-0214149 and the U.S. DOE, Office of Science, under Contract No. W-31-109ENG-38.

\section{APPENDIX: EIGENFUNCTIONS AND EIGENVALUES OF EQUATION (32)}

In this Appendix we find the eigenfunctions of discrete spectrum and the phase shifts of continuous spectrum of equation (32), as well as the respective eigenvalues $\tilde{\lambda}_{i}$. In 
order to solve equation (32) one needs to diagonalize the Hamiltonian

$$
H_{m}=-\frac{d^{2}}{d \xi^{2}}-\frac{m(m+1)}{4 \cosh ^{2}(\xi / 2)}
$$

with $m=3$.

The eigenstates of the Hamiltonian A.1 can be obtained using algebraic technique called supersymmetric quantum mechanics, see Refs. 33 34 35. We begin by introducing the raising and lowering operators

$$
a^{ \pm}=\mp \frac{d}{d \xi}+\frac{m}{2} \tanh \frac{\xi}{2} .
$$

Hamiltonians $H_{m}$ and $H_{m-1}$ can be expressed in terms of $a^{+}$and $a^{-}$as

$$
\begin{aligned}
H_{m} & =a^{+} a^{-}-\frac{m^{2}}{4}, \\
H_{m-1} & =a^{-} a^{+}-\frac{m^{2}}{4} .
\end{aligned}
$$

Let us consider the function $\Psi(\xi)=1 / \cosh ^{m}(\xi / 2)$. It satisfies the condition $a^{-} \Psi(\xi)=0$, and therefore $\Psi$ is an eigenfunction of the Hamiltonian $H_{m}$ with eigenvalue $\epsilon=-m^{2} / 4$. Since $\Psi(\xi)$ has no zeros, it describes the ground state of $H_{m}$. Thus we find the ground-state wave function and energy

$$
\Psi_{0}^{(m)}(\xi)=\sqrt{\frac{\Gamma\left(m+\frac{1}{2}\right)}{2 \sqrt{\pi} \Gamma(m)}} \frac{1}{\cosh ^{m}(\xi / 2)}, \quad \epsilon_{0}^{(m)}=-\frac{m^{2}}{4} .
$$

Applying the raising operator $a^{+}$to $H_{m-1} \Psi_{i}^{(m-1)}=$ $\epsilon_{i}^{(m-1)} \Psi_{i}^{(m-1)}$ we find that $a^{+} \Psi_{i}^{(m-1)}$ are eigenfunctions of Hamiltonian $H_{m}$ with eigenvalues $\epsilon_{i}^{(m-1)}$. Upon appropriate normalization we obtain

$\Psi_{i}^{(m)}(\xi)=\frac{1}{\sqrt{\epsilon_{i-1}^{(m-1)}+m^{2} / 4}} a^{+} \Psi_{i-1}^{(m-1)}, \quad \epsilon_{i}^{(m)}=\epsilon_{i-1}^{(m-1)}$.
Conversely, by acting with $a^{-}$on $H_{m} \Psi_{i}^{(m)}=\epsilon_{i}^{(m)} \Psi_{i}^{(m)}$ with $i>0$, one finds that $a^{-} \Psi_{i}^{(m)}$ are eigenfunctions of Hamiltonian $H_{m-1}$ with eigenvalues $\epsilon_{i}^{(m)}$. Therefore, we conclude that in addition to its ground-state eigenvalue $\epsilon_{0}^{(m)}$ given by (A.4), the spectrum of $H_{m}$ consists of all the eigenvalues of $H_{m-1}$.

Using Eqs. A.4 and A.5 one can derive the eigenfunctions and eigenvalues of $H_{m}$ from those of $H_{m-1}$ and vice versa. To illustrate this, let us consider the Hamiltonian $H_{0}=-(d / d \xi)^{2}$. Its eigenfunctions are $e^{ \pm i q \xi}$, and the spectrum is given by $\epsilon_{q}^{(0)}=q^{2}$, where $q$ is an arbitrary real wave number. Then using Eq. A.5 we can find the eigenstates of Hamiltonian $H_{1}$. The continuous spectrum is obviously $\epsilon_{q}^{(0)}$, and its normalized eigenfunctions are

$$
\Psi_{q}^{(1)}(\xi)=\frac{1}{\sqrt{2 \pi\left(q^{2}+1 / 4\right)}}\left(-\frac{d}{d \xi}+\frac{1}{2} \tanh \frac{\xi}{2}\right) e^{i q \xi}
$$

The ground-state wave function and eigenvalue of $H_{1}$ are given by Eq. A.4 with $m=1$.

Applying the same technique two more times we find the bound states of $H_{3}$,

$$
\begin{aligned}
& \Psi_{0}^{(3)}(\xi)=\frac{\sqrt{15}}{4 \sqrt{2}} \frac{1}{\cosh ^{3}(\xi / 2)}, \quad \epsilon_{0}^{(3)}=-\frac{9}{4} \\
& \Psi_{1}^{(3)}(\xi)=\frac{\sqrt{15}}{2 \sqrt{2}} \frac{\sinh (\xi / 2)}{\cosh ^{3}(\xi / 2)}, \quad \epsilon_{1}^{(3)}=-1 \\
& \Psi_{2}^{(3)}(\xi)=\frac{\sqrt{3}}{\sqrt{2} \cosh \frac{\xi}{2}}-\frac{5 \sqrt{3}}{4 \sqrt{2} \cosh ^{3} \frac{\xi}{2}}, \quad \epsilon_{2}^{(3)}=-\frac{1}{4} .
\end{aligned}
$$

The eigenfunctions of continuous spectrum of $\mathrm{H}_{3}$ are given by

$$
\Psi_{q}^{(3)}(\xi)=\frac{1}{\sqrt{2 \pi\left(q^{2}+9 / 4\right)\left(q^{2}+1\right)\left(q^{2}+1 / 4\right)}}\left(-\frac{d}{d \xi}+\frac{3}{2} \tanh \frac{\xi}{2}\right)\left(-\frac{d}{d \xi}+\tanh \frac{\xi}{2}\right)\left(-\frac{d}{d \xi}+\frac{1}{2} \tanh \frac{\xi}{2}\right) e^{i q \xi}
$$

Noting that $H_{3}$ coincides up to a constant with the Hamiltonian of equation (32), we conclude that $\tilde{\phi}_{i}(\xi)=$ $\Psi_{i}^{(3)}(\xi)$. Therefore the discrete spectrum of Eq. (32) has three eigenvalues,

$$
\tilde{\lambda}_{0}=-\frac{5}{4}, \quad \tilde{\lambda}_{1}=0, \quad \tilde{\lambda}_{2}=\frac{3}{4}
$$

The continuous spectrum of Eq. (32) is $\tilde{\lambda}_{q}=1+q^{2}$. The asymptotics of its eigenfunctions A.10 can be expressed in terms of the scattering phase shifts $\delta(q)$ in the following way

$$
\tilde{\phi}_{q}(\xi) \rightarrow \frac{i \operatorname{sgn} q}{\sqrt{2 \pi}} e^{i q \xi} e^{ \pm i \delta(q)}, \quad \xi \rightarrow \pm \infty .
$$

Here the absence of the reflected wave illustrates the fact 
that the Hamiltonian $H_{m}$ describes scattering in reflectionless potential 36 Comparing Eqs. A.10 and (A.12) one can see that the phase shifts are given by Eq. (34).

For the evaluation of the prefactor of the switching time we need to study a finite system. In Sec. IVC we consider the system of length $2 \Lambda$ with periodic boundary conditions (39). At $\Lambda \gg 1$ the eigenfunctions of discrete spectrum of Eq. (32) with the boundary conditions (39) are given by Eq. A.11. The quasicontinuous spectrum of a large finite system can be obtained by applying periodic boundary conditions (39) to the eigenfunctions $\tilde{\phi}_{q}$ in the asymptotic form A.12). As a result we find the wave number quantization

$$
q_{l}=\frac{\pi l-\delta\left(q_{l}\right)}{\Lambda},
$$

where $l$ is an integer. Because the phase shift (34) is an odd function of $q$, it follows from Eq. A.13 that $q_{-l}=$ $-q_{l}$. Therefore, the eigenfunctions with wave numbers $q_{l}$ and $q_{-l}$ have the same eigenvalue $\tilde{\lambda}=1+q_{l}^{2}$. Thus the quasicontinuous spectrum of Eq. (32) with periodic boundary conditions (39) is doubly degenerate. The two real solutions with the same $\tilde{\lambda}_{q}$ can be represented as even and odd combinations of $\tilde{\phi}_{q}$ and $\tilde{\phi}_{-q}$.

Transcendental equation A.13 can be solved using iteration procedure. At $\Lambda \gg 1$ it is sufficient to perform only the first iteration. Substituting the result into $\tilde{\lambda}_{i}=$ $1+q_{i}^{2}$ we obtain Eq. (33).

In Sec. IVB we consider the system of length $\Lambda$ with the boundary conditions (26). All solutions of this problem can be obtained from the solutions of the problem with the boundary conditions (39). Indeed, any even solution of the eigenvalue problem (32) with the boundary conditions (39) satisfies the conditions (26). Therefore, from Eqs. A.7 -A.9) and A.11 one concludes that the discrete spectrum consists of two eigenvalues $-5 / 4$ and $3 / 4$. Because only even combinations $\tilde{\phi}_{q}(\xi)+\tilde{\phi}_{q}(-\xi) \equiv$ $\tilde{\phi}_{q}(\xi)-\tilde{\phi}_{-q}(\xi)$ satisfy the conditions (26), the quasicontinuous spectrum is nondegenerate and given by Eq. (33).
* Present address: Department of Physics and Astronomy, The Johns Hopkins University, Baltimore, Maryland 21218, USA

1 V. J. Goldman, D. C. Tsui, and J. E. Cunningham, Phys. Rev. Lett. 58, 1256 (1987).

2 E. S. Alves, L. Eaves, M. Henini, O.H. Hughes, M. L. Leadbeater, F. W. Sheard, G. A. Toombs, G. Hill, and M. A. Pate, Electron. Lett. 24, 1190 (1988).

3 A. Zaslavsky, V. J. Goldman, D. C. Tsui, and J. E. Cunningham, Appl. Phys. Lett. 53, 1408 (1988).

${ }^{4}$ R. K. Hayden, L. Eaves, M. Henini, D. K. Maude, and J. C. Portal, Phys. Rev. B 49, 10745 (1994).

5 J. L. Jimenez, E. E. Mendez, X. Li, and W. I. Wang, Phys. Rev. B 52, R5495 (1995).

6 J. Kastrup, H. T. Grahn, K. Ploog, F. Prengel, A. Wacker, and E. Schöll, Appl. Phys. Lett. 65, 1808 (1994).

7 J. Kastrup, F. Prengel, H. T. Grahn, K. Ploog, and E. Schöll, Phys. Rev. B 53, 1502 (1996).

8 K. J. Luo, H. T. Grahn, and K. H. Ploog, Phys. Rev. B 57, R6838 (1998).

9 M. Rogozia, S. W. Teitsworth, H. T. Grahn, and K. H. Ploog, Phys. Rev. B 64, 041308(R) (2001).

10 Ya. M. Blanter and M. Büttiker, Phys. Rev. B 59, 10217 (1999).

11 O. A. Tretiakov, T. Gramespacher, and K. A. Matveev, Phys. Rev. B 67, 073303 (2003).

12 O. A. Tretiakov and K. A. Matveev, Phys. Rev. B 71, 165326 (2005).

13 J. S. Langer, Ann. Phys. (N.Y.) 41, 108 (1967); ibid. 54, 258 (1969).

14 J. S. Langer and V. Ambegaokar, Phys. Rev. 164, 498 (1967).

15 D. E. McCumber and B. I. Halperin, Phys. Rev. B 1, 1054 (1970).

16 J. Kurkijärvi, Phys. Rev. B 6, 832 (1972).

17 S. V. Iordanskii and A. M. Finkel'shtein, Zh. Eksp. Teor. Fiz. 62, 403 (1972) [Sov. Phys. JETP 35, 215 (1972)]; J.
Low Temp. Phys. 10, 423 (1973).

18 A. N. Jordan and E. V. Sukhorukov, Phys. Rev. Lett. 93, 260604 (2004).

19 R. H. Victora, Phys. Rev. Lett. 63, 457 (1989).

20 M. B. Voloshin, I. Yu. Kobzarev, and L. B. Okun', Yad. Fiz. 20, 1229 (1974) [Sov. J. Nucl. Phys. 20, 644 (1975)].

21 S. Coleman, Phys. Rev. D 15, 2929 (1977); 16, 1248(E) (1977).

22 C. G. Callan and S. Coleman, Phys. Rev. D 16, 1762 (1977).

23 V. G. Kiselev and K. G. Selivanov, JETP Lett. 39, 85 (1984).

24 K. G. Selivanov, Zh. Eksp. Teor. Fiz. 94, 57 (1988) [Sov. Phys. JETP 67, 1548 (1988)].

25 M. I. Dykman, E. Mori, J. Ross, and P. M. Hunt, J. Chem. Phys. 100, 5735 (1994).

26 V. Elgart and A. Kamenev, Phys. Rev. E 70, 041106 (2004).

27 In this paper we study the problem with the cubic potential given by Eq. (2). A similar problem was considered in Ref. 37. A one-dimensional decay problem with a quartic potential was addressed in Refs. 38 and 39.

28 I. S. Gradshteyn and I. M. Ryzhik, Table of Integrals, Series, and Products (Academic, New York, 1980).

29 N. G. van Kampen, Stochastic Processes in Physics and Chemistry, 2nd ed. (Elsevier, Amsterdam, 1997).

30 B. J. Matkowsky and Z. Schuss, SIAM J. Appl. Math. 33, 365 (1977).

31 P. Hänggi, P. Talkner, and M. Borkovec, Rev. Mod. Phys. 62, 251 (1990), see Eq. (7.46).

32 By differentiating Eq. (8), one can check explicitly that $z_{s}^{\prime}(\xi)$ is indeed a solution of equation (32) with $\tilde{\lambda}=0$.

33 M. Shifman, A. Vainshtein, and M. Voloshin, Phys. Rev. D 59, 045016 (1999).

34 E. Witten, Nucl. Phys. B 188, 513 (1981); 202, 253 (1982).

35 F. Schwabl, Quantum Mechanics, 2nd ed. (SpringerVerlag, Berlin, Heidelberg, New York, 1995). 
36 L. D. Landau and E. M. Lifshitz, Quantum Mechanics, 3rd ed. (Permagon, Oxford, 1977).

37 M. Büttiker and R. Landauer, Phys. Rev. A 23, 1397 (1981).
38 R. S. Maier and D. L. Stein, Phys. Rev. Lett. 87, 270601 (2001).

39 D. L. Stein, Brazilian J. Phys. 35, 242 (2005). 